\title{
A Yeast-Based Biosensor for Screening of Short- and Medium-Chain Fatty Acid Production
}

\author{
Leonie Baumann, ${ }^{\dagger}$ Arun S. Rajkumar, ${ }^{\dagger}$ John P. Morrissey, ${ }^{\ddagger}$ Eckhard Boles, ${ }^{\dagger}$ and Mislav Oreb*, \\ ${ }^{\dagger}$ Institute of Molecular Biosciences, Faculty of Biological Sciences, Goethe University Frankfurt, Max-von-Laue Straße 9, 60438 \\ Frankfurt am Main, Germany \\ ${ }^{\ddagger}$ School of Microbiology, Centre for Synthetic Biology and Biotechnology, Environmental Research Institute, APC Microbiome \\ Institute, University College Cork, Cork T12 YN60, Ireland
}

Supporting Information

ABSTRACT: Short- and medium-chain fatty acids (SMCFA) are important platform chemicals currently produced from nonsustainable resources. The engineering of microbial cells to produce SMCFA, however, lacks high-throughput methods to screen for best performing cells. Here, we present the development of a whole-cell biosensor for easy and rapid detection of SMCFA. The biosensor is based on a multicopy yeast plasmid containing the SMCFA-responsive PDR12 promoter coupled to GFP as the reporter gene. The sensor detected hexanoic, heptanoic and

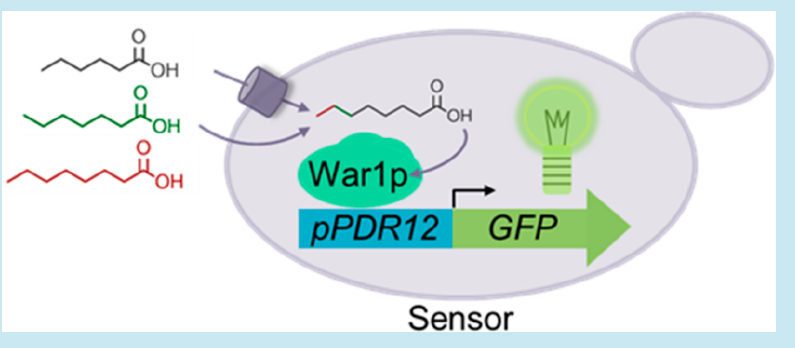
octanoic acid over a linear range up to $2,1.5$, and $0.75 \mathrm{mM}$, respectively, but did not show a linear response to decanoic and dodecanoic acid. We validated the functionality of the biosensor with culture supernatants of a previously engineered Saccharomyces cerevisiae octanoic acid producer strain and derivatives thereof. The biosensor signal correlated strongly with the octanoic acid concentrations as determined by gas chromatography. Thus, this biosensor enables the high-throughput screening of SMCFA producers and has the potential to drastically speed up the engineering of diverse SMCFA producing cell factories.

KEYWORDS: biosensor, high-throughput screening, short-chain fatty acids, medium-chain fatty acids, octanoic acid, PDR12

$S^{\text {thas }}$ hort- and medium-chain fatty acids (SMCFA) and their derivatives have a wide range of industrial applications such as pharmaceuticals, antimicrobials or biofuels. ${ }^{1}$ Rising concerns about nonsustainable production methods, such as deforestation and competition with the food industry, makes the fermentative production from renewable resources by microbial cell factories increasingly attractive. In recent years, several reports about the successful engineering of strains for the production of SMCFA have been published, but yields, titers and productivities remain low. ${ }^{2}$ One of the reasons for this is the lack of high-throughput screening methods for randomly mutated or rationally engineered strains to identify highly producing cells, as the common screening methods currently applied are mostly based on low-throughput gas (GC) or liquid chromatography (LC). ${ }^{3}$

The use of biosensors for screening has become a popular alternative to chromatography and can substantially accelerate the optimization of production strains. ${ }^{4}$ Most developed biosensor systems rely on the expression of sensing elements, e.g., genes encoding extracellular receptors, under the control of an inducible promoter, and are coupled with an easily detectable output such as fluorescence, luminescence, colorimetry or growth rate. 4

To our knowledge only one whole-cell SMCFA biosensor was developed so far, which was also based on this principle. ${ }^{5}$ It made use of heterologous G-protein coupled receptors
(GPCRs) in combination with the yeast mating pathway for signaling and a reporter construct inducing GFP expression upon exposure to octanoic acid $\left(\mathrm{C}_{8} \mathrm{FA}\right)$ and decanoic acid $\left(\mathrm{C}_{10} \mathrm{FA}\right)$. Although this GPCR-based sensor system has the advantage of being amenable to the sensing of other compounds by exchanging the receptor unit, ${ }^{6}$ it has some drawbacks including complicated strain construction (several gene deletions and insertions), the need for functional expression of heterologous plasma membrane localized receptors as well as a low linear range for $\mathrm{C}_{8}$ FA detection. Most importantly, the functionality of this sensor could not be verified in a mixture with other secreted acids, i.e., myristic and palmitic acid $\left(\mathrm{C}_{14}\right.$ and $\left.\mathrm{C}_{16} \mathrm{FA}\right)$, which was partially attributed to the toxicity of FA on cell growth. ${ }^{5}$ However, functionality of a sensor in a mixture of different acids, as found in culture supernatants, would be essential for practical application.

Due to these limitations, we assumed that a promoter-based biosensor for SMCFA detection could be a more promising approach. There are several examples of transcription factor/ promoter-based biosensors developed for the detection of environmental pollutants but not so many for the screening of mutant libraries to identify best performing cells. ${ }^{7-11} \mathrm{~A}$ prerequisite for the use of such systems as biosensors is the

Received: July 20, 2018 
identification of a substrate-specific transcription factor and its respective promoter. ${ }^{12}$ The PDR 12 promoter appeared as a suitable candidate for the sensor system that we envisioned, as it is rapidly activated by only one transcription factor ${ }^{13,14}$ and responds to a narrow spectrum of substrates. ${ }^{13,15-17}$ PDR12 encodes an ATP-binding cassette transporter and is under control of the $\mathrm{Zn}_{2} \mathrm{Cys}_{6}$ transcription factor Warlp. ${ }^{13,14}$ Warlp constitutively binds to a weak acid response element (WARE) in the PDR12 promoter. Upon weak acid stress, Warlp is phosphorylated and undergoes conformational changes leading to its activation and thereby PDR 12 expression. ${ }^{14,18,19}$ PDR12 has been shown so far to be induced by multiple substrates, i.e., propanoic, butanoic, pentanoic, hexanoic and heptanoic acids $\left(\mathrm{C}_{3}-\mathrm{C}_{7} \mathrm{FA}\right), \mathrm{C}_{8} \mathrm{FA},{ }^{15,17,20}$ sorbate and benzoate ${ }^{13}$ but not by acetate, ${ }^{16}$ succinate, citrate ${ }^{17}$ or organic alcohols. ${ }^{15}$ The role of Pdr12p in response to $\mathrm{C}_{3}-\mathrm{C}_{8}$ FA has been demonstrated with a $\Delta p d r 12$ mutant that was hypersensitive and the activation of a PDR12-lacZ reporter construct in response to these FA. ${ }^{15,17}$ Furthermore, a microarray analysis revealed the activation of PDR12 expression upon exposure of yeast cells to $\mathrm{C}_{8} \mathrm{FA}^{20}$ In addition to these characteristics, the PDR12 promoter has a low constitutive activity in the absence of stress, ${ }^{14}$ but upon the appropriate signal exposure, $\operatorname{Pdr} 12 p$ becomes one of the most abundant plasma membrane proteins. ${ }^{21}$

These previous findings led us to the assumption that GFP expression driven by the PDR 12 promoter could be suitable as a biosensor for SMCFA. We further sought to develop it as a two-cell sensor system in which SMCFA sensing is decoupled from SMCFA production for several reasons. First, with increasing titers, the producer microbe supernatant can simply be diluted to stay in the linear range of the sensor, which also helps to evade detrimental effects of SMCFA on yeast growth at higher concentrations. ${ }^{22}$ A one-cell sensor system could lead to false-negatives due to a saturation of the sensor signal at concentrations exceeding the linear range. Second, the producer microbe can be further engineered or modified without affecting the sensor function. Finally, we want to select for cells producing high extracellular titers. In a one-cell system, an intracellular sensor will only measure the cytosolic amount of produced SMCFA, which can lead, for example, to the identification of transport-deficient mutants that accumulate SMCFA intracellularly instead of producing higher titers.

Previously, we engineered an $S$. cerevisiae strain producing mainly $\mathrm{C}_{8}$ FA with titers of up to $245 \mathrm{mg} / \mathrm{L}(1.7 \mathrm{mM})$ and minor amounts of $\mathrm{C}_{6}, \mathrm{C}_{10}$ and $\mathrm{C}_{12} \mathrm{FA}^{23}$ Interested in improving this strain for higher titers, we aimed at developing a biosensor assay for rapid detection of SMCFA in culture supernatants of this strain and mutants thereof.

\section{RESULTS AND DISCUSSION}

\section{Design of a PDR12 Promoter-Based Biosensor for} SMCFA. We sought to develop a two-cell sensor system in which SMCFA sensing is decoupled from SMCFA production as schematically illustrated in Figure 1. The biosensor was designed by fusing the PDR12 promoter (corresponding to 1168 bp upstream of the PDR12 coding sequence) ${ }^{15}$ to the open reading frame of enhanced GFP, namely Envy, ${ }^{24}$ and inserted into a multicopy plasmid that was transformed into $S$. cerevisiae CEN.PK113-11C. The sensor's functionality relies on the uptake of exogenous SMCFA, such as $\mathrm{C}_{8} \mathrm{FA}$, by the yeast cells. ${ }^{25}$ At physiological $\mathrm{pH}, \mathrm{C}_{8}$ FA are taken up via active transport, whereas at acidic $\mathrm{pH}$ the undissociated acid form can enter the cell by passive diffusion. ${ }^{22,26}$ Once taken up by

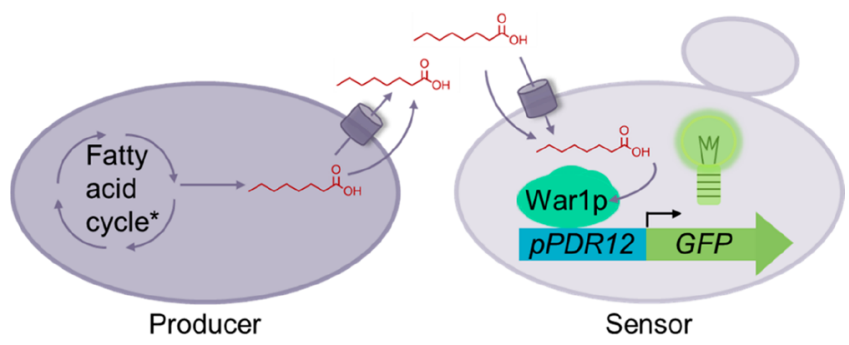

Figure 1. Short- and medium-chain fatty acid detection by a wholecell biosensor. A producer microbe secretes short- and/or mediumchain fatty acids (SMCFA), such as octanoic acid shown here, which are produced through a modified fatty acid cycle $(*)$. The culture supernatant is added to the yeast biosensor strain, which takes up the SMCFA via diffusion or active transport. The biosensor consists of the PDR12 promoter ( $p P D R 12)$ coupled to an enhanced version of green fluorescent protein (GFP). The transcription factor Warlp, which is constitutively bound to $p P D R 12$, undergoes phosphorylation and conformational changes upon signal exposure and initiates the expression of GFP.

the cell, the $\mathrm{C}_{8} \mathrm{FA}$ activates Warlp, leading to transcription from the PDR 12 promoter. We found that GFP expression is activated only in the presence of the inducing signal (here: $\mathrm{C}_{8}$ FA); in the absence of signal, we observed only low constitutive fluorescence (Figure S1). To prove the functionality of the biosensor in culture supernatants, we used a previously constructed $S$. cerevisiae strain (RPY21/FAS ${ }^{\mathrm{R} 1834 \mathrm{~K}}$ ) that produces mainly $\mathrm{C}_{8} \mathrm{FA}$, which is secreted from or diffuses out of the cells. ${ }^{25}$

Characterization and Verification of Functionality of the Biosensor. We analyzed the biosensor response to $\mathrm{C}_{8} \mathrm{FA}$ concentrations between 0 and $1 \mathrm{mM}$ for an initial validation in defined medium (SCD). We observed a correlation of the RFI (relative fluorescence intensity) of the biosensor to $\mathrm{C}_{8} \mathrm{FA}$ over the entire concentration range and a linear response between 0.01 and $0.75 \mathrm{mM}$ (Figure $2 \mathrm{~A}$ ), which is a strong improvement over a previously published GPCR-based $\mathrm{C}_{8}$ FA sensor (0.019-0.25 mM linear range). ${ }^{5}$ The highest intensity of the signal after activation showed a nearly 10-fold increase after $2 \mathrm{~h}$ of incubation with $\mathrm{C}_{8} \mathrm{FA}$, displaying a high dynamic range (Figure 2A, Figure S2). To make sure that the activation of the biosensor is specific, we tested a control strain with a plasmid expressing GFP under control of the MET25 promoter, which is methionine repressible. In this control strain we observed no $\mathrm{C}_{8}$ FA concentration-dependent induction of GFP expression in SCD medium without methionine (Figure 2A).

Industrial microbial fermentations commonly use complex medium rather than defined medium. Therefore, we next tested the biosensor in a complex medium, i.e., YPD. Again, we observed a correlation of the RFI to the tested $\mathrm{C}_{8}$ FA concentrations $(0-3 \mathrm{mM})$ and an identical linear range between 0.01 and $0.75 \mathrm{mM}$ (Figure 2B, Figure S3). While the dynamic range of the sensor was lower than in SCD medium even after twice the incubation time (Figure S4), this can be attributed to the higher background fluorescence of YPD. Nevertheless, the maximum increase in the intensity of the signal after activation was more than tripled after $4 \mathrm{~h}$ of incubation. Even though there is a discrepancy in previous reports about the PDR12 induction by $\mathrm{C}_{8} \mathrm{FA},{ }^{15,17,20}$ we could clearly show that the PDR 12 promoter's $\mathrm{C}_{8}$ FA-dependent response allows it to be used as a biosensor. To our knowledge, this is the first SMCFA biosensor shown to function reliably in 
A

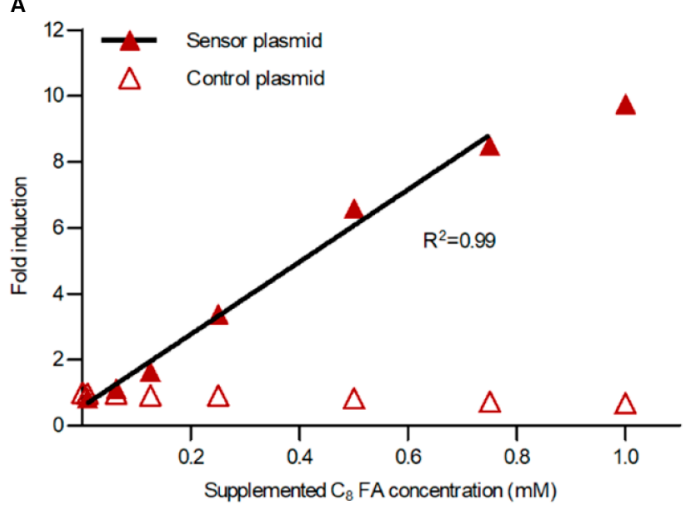

C

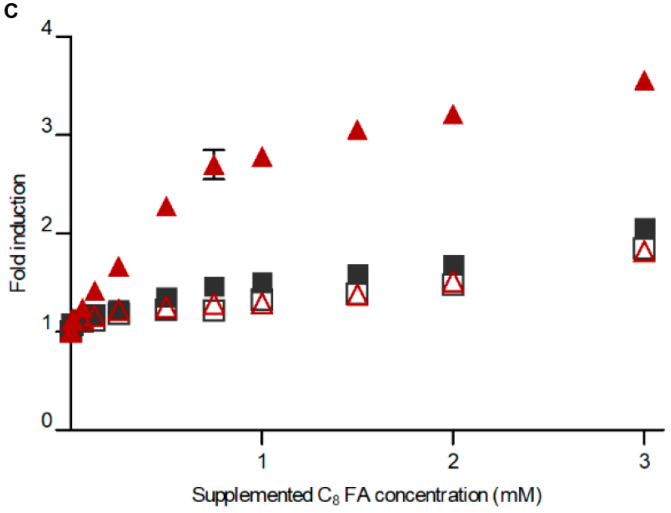

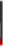

B

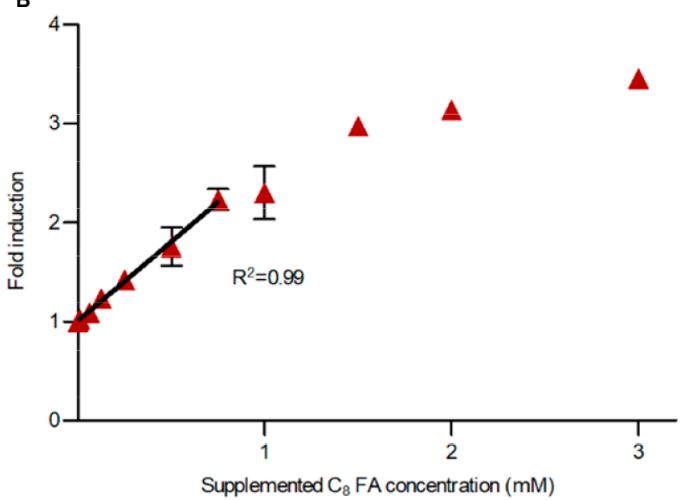

Sensor plasmid

Control plasmid

Sensor genomic

Control genomic

Figure 2. $\mathrm{C}_{8}$ fatty acid-dependent response of plasmid-based and genomically integrated biosensors. Response and linear range of the plasmidbased biosensor after $2 \mathrm{~h}$ incubation with $\mathrm{C}_{8}$ fatty acids (FA) in SCD (A) and after $4 \mathrm{~h}$ in YPD medium (B). Error bars represent two technical replicates. Experiments were conducted in three biological replicates with comparable results (Figure S2, Figure S3). (C) Response of the genomically integrated biosensor after $4 \mathrm{~h}$ incubation with $\mathrm{C}_{8}$ FA in YPD. Sensor plasmid: CEN.PK113-11C + p426pPDR12-GFP. Control plasmid: CEN.PK113-11C + p426pMET25-GFP. Sensor genomic: LBY27. Control genomic: CEN.PK113-11C. For fold induction, fluorescence intensities (FI) were divided by optical densities $\left(\mathrm{OD}_{600}\right)$ and normalized to $\mathrm{FI} / \mathrm{OD}_{600}$ values of samples without $\mathrm{C}_{8} \mathrm{FA}$.

complex medium. This is of particular importance since the highest SMCFA titers produced in yeast so far were reached in complex medium. ${ }^{23,27,28}$

To avoid possible stability problems by using a multicopy plasmid-based biosensor, we integrated the sensor construct into the $S$. cerevisiae CEN.PK113-11C genome resulting in strain LBY27. This sensor strain, however, only showed a barely detectable increase in the intensity of the signal upon $\mathrm{C}_{8}$ FA addition in comparison to the control CEN.PK113-11C strain (Figure 2C). Therefore, a multicopy plasmid seems to be necessary for a good dynamic range, and we continued to work with the plasmid-based biosensor.

The Biosensor Also Responds to $C_{6}$ and $C_{7}$ but Not $\mathrm{C}_{10}$ or $\mathrm{C}_{12}$ FA. It was shown in previous work that PDR12 expression is influenced by several weak acids. ${ }^{13,15-17}$ As none of these compounds-except other SMCFA-are usually produced by $S$. cerevisiae, we reasoned that they should not influence the biosensor's ability to detect SMCFA in $S$. cerevisiae culture supernatants.

The previously engineered $S$. cerevisiae strain RPY21/ FAS ${ }^{\mathrm{R} 1834 \mathrm{~K}}$ mainly produces $\mathrm{C}_{8} \mathrm{FA}$ and in minor amounts $\mathrm{C}_{6}$, $\mathrm{C}_{10}$ and $\mathrm{C}_{12}$ FA. To test whether the biosensor also detects these byproducts, we analyzed its response upon addition of $\mathrm{C}_{6}, \mathrm{C}_{10}$ and $\mathrm{C}_{12} \mathrm{FA}$ to YPD. As depicted in Figure 3, the RFI of the biosensor correlates to $C_{6}$ FA levels between 0 and $3 \mathrm{mM}$, and the biosensor showed a linear response between 0.01 and $2 \mathrm{mM}$ in all replicates (Figure S3). $\mathrm{C}_{10}$ FA seems to be very toxic to the cells, as growth is already strongly decreased at the

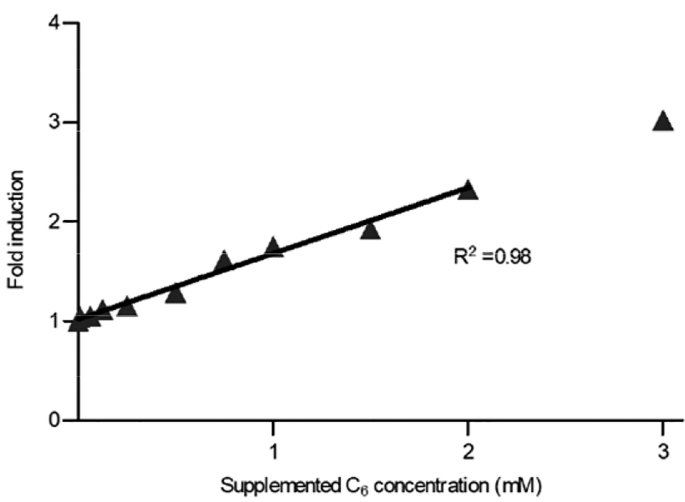

Figure 3. $\mathrm{C}_{6}$ fatty acid-dependent response of the biosensor. Response and linear range of the biosensor after $4 \mathrm{~h}$ incubation with $\mathrm{C}_{6}$ fatty acids (FA) in YPD medium. Error bars represent two technical replicates. Experiments were conducted in three biological replicates with comparable results (Figure S3). For fold induction, fluorescence intensities (FI) were divided by optical densities $\left(\mathrm{OD}_{600}\right)$ and normalized to $\mathrm{FI} / \mathrm{OD}_{600}$ values of samples without FA.

lower concentrations, which leads to false high inductions when dividing FI by $\mathrm{OD}_{600}$ values (Figure S3). This is in accordance with previous findings about high toxicity of $\mathrm{C}_{10}$ FA. $^{22}$ Overall, we cannot exclude a very slight response of the biosensor to $\mathrm{C}_{10} \mathrm{FA}$ at low concentrations $(0.01-0.25 \mathrm{mM})$, which would be in accordance to a previous report suggesting a discrete role of Pdr12p in $\mathrm{C}_{10}$ FA response. ${ }^{17}$ Nevertheless, we 
A

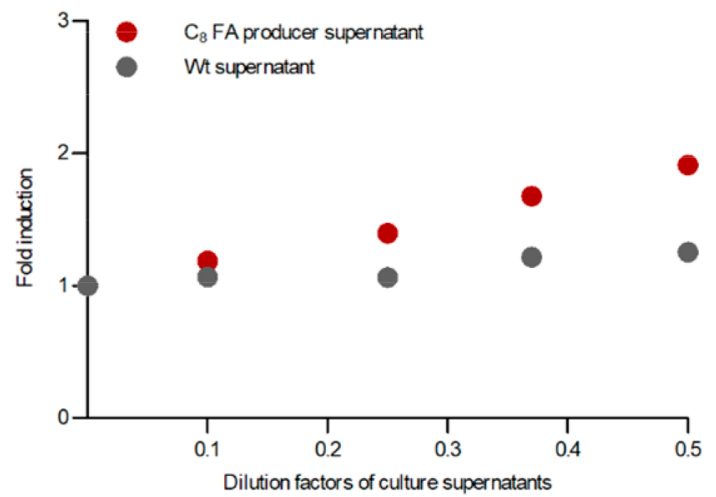

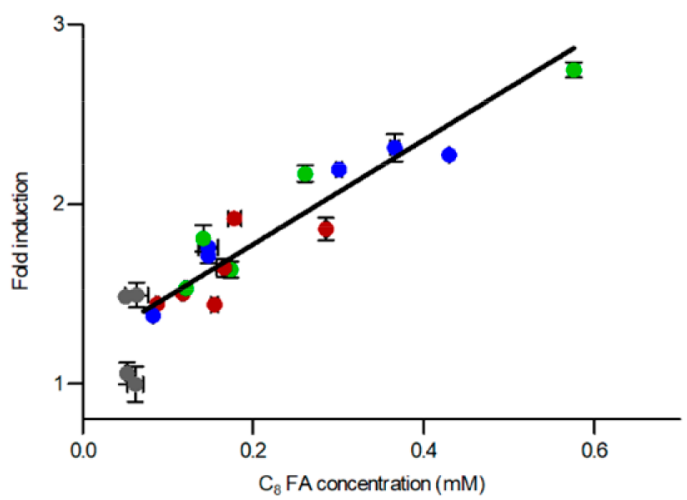

Figure 4. Biosensor response to $\mathrm{C}_{8}$ fatty acids in S. cerevisiae culture supernatants and correlation to GC measurement. (A) Response of the biosensor to differently diluted culture supernatants of a $\mathrm{C}_{8}$ fatty acid (FA) producer (RPY21/FAS $\left.{ }^{\mathrm{R} 1834 \mathrm{~K}} / \mathrm{pRS} 42 \mathrm{H}\right)$ and a wildtype strain (Wt, CEN.PK113-7D). (B) Linear correlation of the fold induction of biosensor signal in 0.5 dilutions of culture supernatants with GC measurements of the same supernatants. Strains: CEN.PK113-7D (gray), RPY21/FAS R1834K/pRS42H (red), RPY21/FAS ${ }^{\mathrm{R} 1834 \mathrm{~K}} / \mathrm{LBV}^{\mathrm{R}} 17$ (blue), RPY21/ $\mathrm{FAS}^{\mathrm{R} 1834 \mathrm{~K}} / \mathrm{LBV} 20$ (green).

could not observe a linear response range of the biosensor to $\mathrm{C}_{10} \mathrm{FA}$ in all three biological replicates.

For $\mathrm{C}_{12} \mathrm{FA}$, we found a concentration-dependent decrease in fluorescence over the entire concentration range tested and an increase in the $\mathrm{OD}_{600}$ at $\mathrm{C}_{12} \mathrm{FA}$ concentrations between 0.25 and $1 \mathrm{mM}$ (Figure S5). We also observed this when adding $\mathrm{C}_{12} \mathrm{FA}$ to plain medium not containing the biosensor strain (data not shown). Therefore, this is probably due to incomplete solubility and light scattering by $\mathrm{C}_{12}$ FA micelles. In addition, we also tested the biosensor response to the odd chain $\mathrm{C}_{7} \mathrm{FA}$ and observed a linear response between 0.01 and $1.5 \mathrm{mM}$ (Figure S6). This is in accordance to previous studies reporting that PDR12 is induced by $\mathrm{C}_{7} \mathrm{FA}^{15,17}$

In summary, we show that the biosensor responds to $\mathrm{C}_{6}, \mathrm{C}_{7}$ and $\mathrm{C}_{8} \mathrm{FA}$ in YPD medium over linear ranges from 0.01 up to $2,1.5$, and $0.75 \mathrm{mM}$, respectively, whereas it does not show a linear response to $\mathrm{C}_{10} \mathrm{FA}$ and no concentration-dependent response at all to $\mathrm{C}_{12} \mathrm{FA}$.

Detection of $\mathrm{C}_{8} \mathrm{FA}$ by the Biosensor in $\mathrm{S}$. cerevisiae Culture Supernatants. To validate that the biosensor can discriminate between different SMCFA concentrations in culture supernatants, we first conducted a pretest. We cultivated a $\mathrm{C}_{8}$ FA producer strain (RPY21/FAS ${ }^{\mathrm{R} 1834 \mathrm{~K}} /$ pRS42H) and a wildtype strain (CEN.PK113-7D) in buffered YPD (with hygromycin if applicable) for $72 \mathrm{~h}$ and added the culture supernatants in different dilutions to the biosensor, and in parallel we measured the $\mathrm{C}_{8}$ FA content by GC. As shown in Figure $4 \mathrm{~A}$, the lowest dilution $(0.5)$ of the $C_{8}$ FA producer supernatant led to a 1.9-fold increase in the intensity of the signal after $4 \mathrm{~h}$ of incubation. Furthermore, we observed a constant increase in signal intensity with decreasing dilution factors confirming the $\mathrm{C}_{8}$ FA concentration-dependent activation of the biosensor in S. cerevisiae culture supernatants. The supernatant of the wildtype culture also led to a slight increase in signal intensity of the biosensor, which can be attributed to minor amounts of $\mathrm{C}_{8} \mathrm{FA}$ that it produced naturally. As determined by GC, the wildtype strain produced $0.02 \mathrm{mM} \mathrm{C}_{8}$ FA, whereas RPY21/FAS $\mathrm{R}^{\mathrm{R} 1834 \mathrm{~K}} / \mathrm{pRS} 42 \mathrm{H}$ produced $0.28 \mathrm{mM} \mathrm{C}_{8} \mathrm{FA}$.

To obtain supernatants with a wide range of $\mathrm{C}_{8} \mathrm{FA}$ concentrations we not only tested the original RPY21/ $\mathrm{FAS}^{\mathrm{R} 1834 \mathrm{~K}}$ strain but also two derivatives thereof. We transformed the strain with one of two plasmids containing overexpressed genes or the control plasmid (pRS42H), respectively. One plasmid harbored an overexpression construct of TPO1 (LBV17). The transporter Tpolp seems to be involved in the resistance to $\mathrm{C}_{8} \mathrm{FA}^{20}$ and therefore has the potential to decrease $\mathrm{C}_{8}$ FA toxicity. The second construct encoded the acetyl-CoA carboxylase ACC1 and harbored mutations leading to amino acid exchanges of two serine residues to alanine $\left(A C C 1^{\text {S659AS1157A }}\right.$ LBV20). These two mutations impair the deactivation of Acclp via phosphorylation. $^{29-31}$ A similarly modified Acclp $\left(A C C 1^{S 1157 A}\right)$ was shown to have a positive effect on yeast resistance to $\mathrm{C}_{8} \mathrm{FA}{ }^{32}$ and therefore we reasoned that it could also influence $\mathrm{C}_{8} \mathrm{FA}$ product titers of RPY21/FAS ${ }^{\mathrm{R} 1834 \mathrm{~K}}$. We cultivated RPY21/ $\mathrm{FAS}^{\mathrm{R} 1834 \mathrm{~K}}$ with overexpression or control plasmids as well as a wildtype strain (CEN.PK113-7D) in two independent experiments in two to three replicates and obtained a wide range of $\mathrm{C}_{8}$ FA titers between 0.05 and $0.58 \mathrm{mM}$ as measured by GC. We added the culture supernatants to the biosensor (corresponding to a 0.5 dilution) and measured the RFI. As shown in Figure 4B, the RFI of the biosensor clearly correlates with the $\mathrm{C}_{8}$ FA concentrations measured by GC. A linear correlation could be observed for concentrations of produced $\mathrm{C}_{8}$ FA from $0.08 \mathrm{mM}$ up to the highest concentration tested, $0.58 \mathrm{mM}$. We additionally tested the supernatants in a dilution of 0.25 with the biosensor and received-despite lower fold inductions - an identical linear range (Figure S7A). In all samples, the $\mathrm{C}_{6} \mathrm{FA}$ content was below $6 \%$ of the total SMCFA titers and did not alter the linear correlation of the biosensor response to $\mathrm{C}_{8} \mathrm{FA}$ concentrations (Figure S7B).

This is the first report of a whole-cell promoter-based biosensor for the detection of SMCFA in culture supernatants. In contrast to previously generated sensor systems, ${ }^{5,6,8-10}$ the here presented biosensor solely consists of an $S$. cerevisiae strain transformed with a multicopy plasmid containing the PDR12 promoter coupled to GFP without further strain engineering, fine-tuning or expression of additional components needed. We demonstrate that it linearly responds to $\mathrm{C}_{8} \mathrm{FA}$ in defined and complex medium as well as to $\mathrm{C}_{6}$ and $\mathrm{C}_{7} \mathrm{FA}$ in complex medium. Furthermore, the biosensor responds to $\mathrm{C}_{8} \mathrm{FA}$ concentrations from 0.08 up to at least $0.58 \mathrm{mM}$ in $S$. cerevisiae culture supernatants and therefore in a mixture of other secreted FA. The biosensor response showed a clear correlation of RFI to $\mathrm{C}_{8}$ FA values determined by $\mathrm{GC}$ and 
therefore can be used to compare SMCFA titers of different culture supernatants. On the basis of these results, we envision two possible applications. It can be used as a screening system of microbial producer strain libraries of one specific SMCFA, as shown for the $\mathrm{C}_{8} \mathrm{FA}$ producing strain here, or to screen for increased overall SMCFA titers. For the latter, GC measurements can be used to determine the exact SMCFA composition of the culture supernatants once a biosensoraided screening resulted in several good performing candidate strains.

The currently produced amounts of $\mathrm{C}_{6}$ FA by yeast vary from 0.17 to $0.62 \mathrm{mM}^{23,28,33}$ and fall within the linear range of the biosensor. On the other hand, $\mathrm{C}_{8} \mathrm{FA}$ titers produced by yeast vary from 0.45 to $1.7 \mathrm{mM}^{23,28,33}$ and therefore partly exceed the linear range of the biosensor. However, we can simply dilute culture supernatants before adding them to the biosensor to retain a linear response. The biosensor also has a wide linear range for $\mathrm{C}_{7} \mathrm{FA}$ detection and could be a valuable tool to screen a $\mathrm{C}_{7} \mathrm{FA}$-producer library; however, yeast has not been engineered to produce $\mathrm{C}_{7} \mathrm{FA}$ so far.

For future use, a more stable, plasmid-independent sensor strain might be beneficial. This could be achieved by a multicopy integration of the sensor into the genome. Thereby, the low dynamic range of the sensor response as seen with the single genomic integration might be increased. The dynamic range of the sensor could be further optimized by the genomic replacement of the native WAR1 promoter with the PDR12 promoter. ${ }^{11}$ Assuming low "leaky" expression levels of WAR1, such a positive-feedback loop would accelerate WAR1 expression in the presence of inducing molecules. These higher Warlp levels would then result in a higher expression of PDR12 promoter-coupled GFP, possibly leading to higher dynamic ranges of the sensor. To improve the linear range of the biosensor, the saturation of the sensor signal would have to be shifted toward higher FA concentrations. One possibility to achieve this could be to decrease the binding affinity of Warlp to the WARE site in the PDR12 promoter. This might be accomplished either by mutating WAR1 or the WARE site. Alternatively, Warlp could be mutated to exhibit a lower binding affinity for its inducers; however, it is known neither if the inducing molecules directly bind Warlp nor at which molecular site. ${ }^{14,18,19}$ In order to try any of these approaches, detailed Warlp structural data would be needed.

Nevertheless, the here presented biosensor has the appropriate linear and dynamic ranges to use it for highthroughput screening of yeast SMCFA production. Needing only $4 \mathrm{~h}$ of incubation of sample supernatants with the biosensor in multiwell format, hundreds of strains can be rapidly screened to select the best performing strains. For future use, we envision a broader application spectrum of the biosensor as its use is not restricted per se to S. cerevisiae or even yeast SMCFA producers. The biosensor's SMCFA response certainly will have to be confirmed in the supernatants of other microbes, i.e., other yeasts or bacteria, as different species also produce different metabolites that could influence the biosensor's response to SMCFA in other culture supernatants. Overall, this biosensor represents a valuable and ready-to-use system, which fills a need to rapidly improve microbial SMCFA production.

\section{METHODS}

Strains and Plasmid Construction. Yeast strains and plasmids used throughout this study are listed in Table S1. The
PDR12 promoter, TPO1 and $A C C 1^{\text {S659AS1157A }}$ as well as promoters ( $p P G K 1, p P Y K 1)$ and endogenous terminators (tTPO1, tACC1) were amplified from CEN.PK113-11C genomic DNA with primers containing the respective overhangs for cloning via homologous recombination (oligonucleotides are listed in Table S2). The PDR12 promoter was integrated into the SacI site of p426pMET25-GFP, thereby replacing $p M E T 25$. The genomic insertion of $p P D R 12-G F P$ was performed via CRISPR/Cas9 as described previously. ${ }^{34}$ The CRISPR/Cas9 plasmid was amplified in two PCR fragments and transformed for in vivo assembly. The insertion fragment was amplified from plasmid p426pPDR12-GFP. Yeast transformations were performed according to Gietz and Schiestl ${ }^{35}$ or for RPY21 via an adapted procedure. ${ }^{23}$ To generate RPY21/FAS ${ }^{\mathrm{R} 1834 \mathrm{~K}}$, RPY21 was transformed with a plasmid carrying the wildtype version of FAS2 and another plasmid carrying FAS1 with mutations leading to an amino acid replacement $(\mathrm{R} 1834 \mathrm{~K})$, thereby redirecting the FA production from long chain FA to SMCFA. ${ }^{23}$ Cells were streaked out on selective YPD (1\% yeast extract, 2\% peptone, both produced by BD, Difco Laboratories, Sparks, USA; $2 \%$ Dglucose, purchased from Roth, Karlsruhe, Germany) containing hygromycin or G418 $(200 \mu \mathrm{g} / \mathrm{mL})$ to select for $h p h N T 1$ or kanMX or on selective SCD medium ${ }^{36}$ lacking leucine, tryptophan, uracil and/or histidine ( $\pm \mathrm{LWUH})$ to select for LEU2, URA3 or HIS3 markers, respectively. Electrocompetent E. coli DH10 $\beta$ (Gibco BRL, Gaithersburg, MD) was used for subcloning according to standard procedures, and transformants were selected on lysogeny broth (LB) agar plates ${ }^{37}$ supplemented with $100 \mu \mathrm{g} / \mathrm{mL}$ ampicillin.

Cultures for Fatty Acid Production. $\mathrm{C}_{8}$ FA producer and control (wildtype) strains were grown as previously described $^{23}$ with minor adjustments. For precultures, several colonies of the strains were picked and combined in $20 \mathrm{~mL}$ YPD with $100 \mathrm{mM}$ potassium phosphate buffer ( $\mathrm{pH}$ 6.5). After shaking at 180 r.p.m. at $30{ }^{\circ} \mathrm{C}$ overnight, the main culture was inoculated to an $\mathrm{OD}_{600}$ of 0.1 in $30 \mathrm{~mL}$ buffered YPD medium and cultured in $300 \mathrm{~mL}$ shake flasks under the same conditions. After $72 \mathrm{~h}$, the cultures were harvested by centrifugation and $20 \mathrm{~mL}$ of the supernatant was used for FA extraction (see below) whereas remaining supernatant was used for the biosensor assay.

Fatty Acid Extraction and Quantification via GC. FA extraction and GC analysis were performed as described previously. ${ }^{25}$ For each culture, two $10 \mathrm{~mL}$ aliquots from the same culture supernatant were separately processed and measured by GC. The standard deviation (SD) between the two measurements from the same culture was for all samples below $2 \mathrm{mg} / \mathrm{L}$.

Cultures for the Biosensor Assay. For the biosensor assay, strains (CEN.PK113-11C/p426pMET25-GFP or p426pPDR12-GFP) were grown overnight with 180 r.p.m. shaking at $30{ }^{\circ} \mathrm{C}$ in SCD-L-W-U and under addition of repressive concentrations of methionine $(2 \mathrm{mM})$ for the strain harboring plasmid p426pMET25-GFP. Precultured cells were used to inoculate $20 \mathrm{~mL}$ of fresh SCD-L-W-U (+2 $\mathrm{mM}$ methionine) to an $\mathrm{OD}_{600}$ of 0.1 and grown for 4-6 h until an $\mathrm{OD}_{600}$ of about 0.4 . Cells were centrifuged and resuspended in fresh YPD or SCD-L-W-U without methionine and transferred into black 96-well plates with clear flat bottom (ref. 655097, Greiner Bio-one, Frickenhausen, Germany) with $100 \mu \mathrm{L}$ per well. To keep conditions sterile, well plates were sealed with a gas permeable sealing membrane (Breathe-Easy, Diversified 
Biotech, Dedham, MA, USA). For the primary verification of the sensor, $90 \mu \mathrm{L}$ of fresh media was added together with 10 $\mu \mathrm{L}$ of $70 \% \mathrm{v} / \mathrm{v}$ ethanol (control) or $10 \mu \mathrm{L}$ of differently diluted fatty acids (in $70 \% \mathrm{v} / \mathrm{v}$ ethanol) to always reach a final volume of $200 \mu \mathrm{L} /$ well and have the same ethanol concentrations in all wells. $\mathrm{C}_{6}, \mathrm{C}_{7}, \mathrm{C}_{8}, \mathrm{C}_{10}$ and $\mathrm{C}_{12} \mathrm{FA}$ (Sigma-Aldrich) were diluted in $70 \% \mathrm{v} / \mathrm{v}$ ethanol in concentrations between 0.2 and $60 \mathrm{mM}$ to reach final dilutions of 0.01 to $3 \mathrm{mM}$ in the wells. For the biosensor test with culture supernatants, $100 \mu \mathrm{L}$ of culture supernatant (for cultivation conditions see above) was added to $100 \mu \mathrm{L}$ of the biosensor strain. Experiments were performed in biological triplicates with technical duplicates.

Biosensor Measurements. Directly after pipetting of all components into the 96 -well plate, it was incubated at $30{ }^{\circ} \mathrm{C}$ and 600 r.p.m. shaking in a CLARIOstar plate reader (BMG Labtech, Ortenberg, Germany). Measurements of $\mathrm{OD}_{600}$ and FI with excitation at $470 \pm 15 \mathrm{~nm}$ and emission at $515 \pm 20$ $\mathrm{nm}$ were taken every $30 \mathrm{~min}$ over several hours. To normalize for different cell numbers in wells, the FI value of each well was divided by the respective $\mathrm{OD}_{600}$ value. For calculating the fold induction, these $\mathrm{FI} / \mathrm{OD}_{600}$ values were divided for the different $\mathrm{FA}$ concentrations by the $\mathrm{FI} / \mathrm{OD}_{600}$ values of the " $0 \mathrm{mM}$ " controls (without FA). For calculation of the fold induction response to the culture supernatants, $\mathrm{FI} / \mathrm{OD}_{600}$ values of the wells with supernatant and biosensor were divided by FI/ $\mathrm{OD}_{600}$ values only containing biosensor. Values are shown as mean \pm the standard deviation (SD).

Data analyses including the calculation of linear regression and $R^{2}$ values were performed using the software Prism 5 (GraphPad, USA).

\section{ASSOCIATED CONTENT}

\section{S Supporting Information}

The Supporting Information is available free of charge on the ACS Publications website at DOI: 10.1021/acssynbio. 8 b00309.

Supporting figures and tables (PDF)

\section{AUTHOR INFORMATION}

\section{Corresponding Author}

*E-mail:m.oreb@bio.uni-frankfurt.de.

\section{ORCID}

Leonie Baumann: 0000-0001-7579-6777

\section{Author Contributions}

LB, EB and MO conceived the study. ASR and JPM provided ideas and constructive input. LB performed all experiments. LB, EB and MO analyzed the data and wrote the paper. All authors read and approved the manuscript.

\section{Notes}

The authors declare the following competing financial interest(s): EB is inventor of EP patent application No. 15 162192.7 filed on April 1, 2015, and of EP patent application No. 15174342.4 filed on June 26, 2015, by Goethe-University Frankfurt, concerning short-chain acyl-CoA producing FAS variants. There are no other competing interests.

\section{ACKNOWLEDGMENTS}

The authors thank Joanna Tripp for plasmid p426pMET25GFP. We thank Michael K. Jensen and Jay D. Keasling for providing advice and resources. This project has received funding from the European Union's Horizon 2020 research and innovation program under Grant Agreement No. 720824.

\section{ABBREVIATIONS}

SMCFA, short- and medium-chain fatty acid(s); FA, fatty $\operatorname{acid}(\mathrm{s}) ; \mathrm{C}_{6}, \mathrm{C}_{7}, \mathrm{C}_{8}, \mathrm{C}_{10}, \mathrm{C}_{12} \mathrm{FA}$, hexanoic, heptanoic, octanoic, decanoic, dodecanoic acid(s); FAS, fatty acid synthase; PDR12, plasma membrane ATP-binding cassette transporter; TPO1, polyamine transporter of the major facilitator superfamily; ACC1, acetyl-CoA carboxylase; GFP, green fluorescent protein; GC, gas chromatography; $\mathrm{OD}_{600}$, optical density at $\lambda$ $=600 \mathrm{~nm}$; FI, fluorescence intensity; RFI, relative fluorescence intensity.

\section{REFERENCES}

(1) Sarria, S., Kruyer, N. S., and Peralta-Yahya, P. (2017) Microbial synthesis of medium-chain chemicals from renewables. Nat. Biotechnol. 35, 1158-1166.

(2) Fernandez-Moya, R., and Da Silva, N. A. (2017) Engineering Saccharomyces cerevisiae for High-Level Synthesis of Fatty Acids and Derived Products. FEMS Yeast Res., DOI: 10.1093/femsyr/fox071.

(3) Woolston, B. M., Edgar, S., and Stephanopoulos, G. (2013) Metabolic Engineering: Past and Future. Annu. Rev. Chem. Biomol. Eng. 4, 259-288.

(4) Adeniran, A., Sherer, M., and Tyo, K. E. J. (2014) Yeast-based biosensors: design and applications. FEMS Yeast Res. 15, 1-15.

(5) Mukherjee, K., Bhattacharyya, S., and Peralta-Yahya, P. (2015) GPCR-Based Chemical Biosensors for Medium-Chain Fatty Acids. ACS Synth. Biol. 4, 1261-1269.

(6) Ehrenworth, A. M., Claiborne, T., and Peralta-Yahya, P. (2017) Medium-Throughput Screen of Microbially Produced Serotonin via a G-Protein-Coupled Receptor-Based Sensor. Biochemistry 56, 54715475.

(7) Dietrich, J. A., McKee, A. E., and Keasling, J. D. (2010) HighThroughput Metabolic Engineering: Advances in Small-Molecule Screening and Selection. Annu. Rev. Biochem. 79, 563-590.

(8) Skjoedt, M. L., Snoek, T., Kildegaard, K. R., Arsovska, D., Eichenberger, M., Goedecke, T. J., Rajkumar, A. S., Zhang, J., Kristensen, M., Lehka, B. J., Siedler, S., Borodina, I., Jensen, M. K., and Keasling, J. D. (2016) Engineering prokaryotic transcriptional activators as metabolite biosensors in yeast. Nat. Chem. Biol. 12, 951958.

(9) Schuller, A., Rödel, G., and Ostermann, K. (2017) Tuning the Sensitivity of the PDR5 Promoter-Based Detection of Diclofenac in Yeast Biosensors. Sensors 17, 1506.

(10) Siedler, S., Khatri, N. K., Zsohár, A., Kjærbølling, I., Vogt, M., Hammar, P., Nielsen, C. F., Marienhagen, J., Sommer, M. O. A., and Joensson, H. N. (2017) Development of a Bacterial Biosensor for Rapid Screening of Yeast $p$-Coumaric Acid Production. ACS Synth. Biol. 6, 1860-1869.

(11) Williams, T. C., Xu, X., Ostrowski, M., Pretorius, I. S., and Paulsen, I. T. (2017) Positive-feedback, ratiometric biosensor expression improves high-throughput metabolite-producer screening efficiency in yeast. Synth. Biol., DOI: 10.1093/synbio/ysw002.

(12) Michener, J. K., Thodey, K., Liang, J. C., and Smolke, C. D. (2012) Applications of genetically-encoded biosensors for the construction and control of biosynthetic pathways. Metab. Eng. 14, 212-222.

(13) Piper, P., Mahé, Y., Thompson, S., Pandjaitan, R., Holyoak, C., Egner, R., Mühlbauer, M., Coote, P., and Kuchler, K. (1998) The pdr12 $\mathrm{ABC}$ transporter is required for the development of weak organic acid resistance in yeast. EMBO J. 17, 4257-4265.

(14) Kren, A., Mamnun, Y. M., Bauer, B. E., Schüller, C., Wolfger, H., Hatzixanthis, K., Mollapour, M., Gregori, C., Piper, P., and Kuchler, K. (2003) Warlp, a Novel Transcription Factor Controlling Weak Acid Stress Response in Yeast. Mol. Cell. Biol. 23, 1775-1785. 
(15) Hatzixanthis, K., Mollapour, M., Seymour, I., Bauer, B. E., Krapf, G., Schüller, C., Kuchler, K., and Piper, P. W. (2003) Moderately lipophilic carboxylate compounds are the selective inducers of the Saccharomyces cerevisiae Pdr12p ATP-binding cassette transporter. Yeast 20, 575-585.

(16) Bauer, B. E., Rossington, D., Mollapour, M., Mamnun, Y., Kuchler, K., and Piper, P. W. (2003) Weak organic acid stress inhibits aromatic amino acid uptake by yeast, causing a strong influence of amino acid auxotrophies on the phenotypes of membrane transporter mutants. Eur. J. Biochem. 270, 3189-3195.

(17) Holyoak, C. D., Bracey, D., Piper, P. W., Kuchler, K., and Coote, P. J. (1999) The Saccharomyces cerevisiae weak-acid-inducible $\mathrm{ABC}$ transporter Pdr12 transports fluorescein and preservative anions from the cytosol by an energy-dependent mechanism. J. Bacteriol. 181, 4644-4652.

(18) Gregori, C., Schüller, C., Frohner, I. E., Ammerer, G., and Kuchler, K. (2008) Weak organic acids trigger conformational changes of the yeast transcription factor Warl in vivo to elicit stress adaptation. J. Biol. Chem. 283, 25752-64.

(19) Frohner, I. E., Gregori, C., Anrather, D., Roitinger, E., Schü, C., Ammerer, G., and Kuchler, K. (2010) Weak Organic Acid Stress Triggers Hyperphosphorylation of the Yeast Zinc-Finger Transcription Factor Warl and Dampens Stress Adaptation. OMICS 14, $575-586$.

(20) Legras, J. L., Erny, C., Le Jeune, C., Lollier, M., Adolphe, Y., Demuyter, C., Delobel, P., Blondin, B., and Karst, F. (2010) Activation of two different resistance mechanisms in Saccharomyces cerevisiae upon exposure to octanoic and decanoic acids. Appl. Environ. Microbiol. 76, 7526-7535.

(21) Piper, P., Calderon, C. O., Hatzixanthis, K., and Mollapour, M. (2001) Weak acid adaptation: the stress response that confers yeasts with resistance to organic acid food preservatives. Microbiology 147, $2635-2642$.

(22) Borrull, A., López-Martínez, G., Poblet, M., Cordero-Otero, R., and Rozès, N. (2015) New insights into the toxicity mechanism of octanoic and decanoic acids on Saccharomyces cerevisiae. Yeast 32, 451-460.

(23) Gajewski, J., Pavlovic, R., Fischer, M., Boles, E., and Grininger, M. (2017) Engineering fungal de novo fatty acid synthesis for short chain fatty acid production. Nat. Commun. 8, 14650.

(24) Slubowski, C. J., Funk, A. D., Roesner, J. M., Paulissen, S. M., and Huang, L. S. (2015) Plasmids for C-terminal tagging in Saccharomyces cerevisiae that contain improved GFP proteins, Envy and Ivy. Yeast 32, 379-387.

(25) Henritzi, S., Fischer, M., Grininger, M., Oreb, M., and Boles, E. (2018) An engineered fatty acid synthase combined with a carboxylic acid reductase enables de novo production of 1-octanol in Saccharomyces cerevisiae. Biotechnol. Biofuels 11, 150.

(26) Viegas, C. A., and Correia, I. S. (1997) Effects of low temperatures $\left(9-33{ }^{\circ} \mathrm{C}\right)$ and $\mathrm{pH}(3.3-5.7)$ in the loss of Saccharomyces cerevisiae viability by combining lethal concentrations of ethanol with octanoic and decanoic acids. Int. J. Food Microbiol. 34, 267-277.

(27) Leber, C., and Da Silva, N. A. (2014) Engineering of Saccharomyces cerevisiae for the synthesis of short chain fatty acids. Biotechnol. Bioeng. 111, 347-358.

(28) Leber, C., Choi, J. W., Polson, B., and Da Silva, N. A. (2016) Disrupted short chain specific $\beta$-oxidation and improved synthase expression increase synthesis of short chain fatty acids in Saccharomyces cerevisiae. Biotechnol. Bioeng. 113, 895-900.

(29) Choi, J. W., and Da Silva, N. A. (2014) Improving polyketide and fatty acid synthesis by engineering of the yeast acetyl-CoA carboxylase. J. Biotechnol. 187, 56.

(30) Woods, A., Munday, M. R., Scott, J., Yang, X., Carlson, M., and Carling, D. (1994) Yeast SNF1 is functionally related to mammalian AMP-activated protein kinase and regulates acetyl-CoA carboxylase in vivo. J. Biol. Chem. 269, 19509-19515.
(31) Shi, S., Chen, Y., Siewers, V., and Nielsen, J. (2014) Improving production of malonyl coenzyme A-derived metabolites by abolishing Snf1-dependent regulation of Acc1. mBio 5, e01130-14.

(32) Besada-Lombana, P. B., Fernandez-Moya, R., Fenster, J., and Da Silva, N. A. (2017) Engineering Saccharomyces cerevisiae fatty acid composition for increased tolerance to octanoic acid. Biotechnol. Bioeng. 114, 1531-1538.

(33) Zhu, Z., Zhou, Y. J., Krivoruchko, A., Grininger, M., Zhao, Z. K., and Nielsen, J. (2017) Expanding the product portfolio of fungal type I fatty acid synthases. Nat. Chem. Biol. 13, 360-362.

(34) Generoso, W. C., Gottardi, M., Oreb, M., and Boles, E. (2016) Simplified CRISPR-Cas genome editing for Saccharomyces cerevisiae. J. Microbiol. Methods 127, 203-205.

(35) Gietz, R. D., and Schiestl, R. H. (2007) High-efficiency yeast transformation using the LiAc/SS carrier DNA/PEG method. Nat. Protoc. 2, 31-34.

(36) Bruder, S., Reifenrath, M., Thomik, T., Boles, E., and Herzog, K. (2016) Parallelised online biomass monitoring in shake flasks enables efficient strain and carbon source dependent growth characterisation of Saccharomyces cerevisiae. Microb. Cell Fact. 15, 115 .

(37) Sambrook, J., Fritsch, E., and Maniatis, T. (1989) Molecular Cloning: A Laboratory Manual, 2nd ed., Cold Spring Harbor Laboratory Press, Cold Spring Harbor, NY. 